\title{
Problemas de salud mental relacionados con la COVID-19 en el personal de salud y la población general del Perú.
}

\begin{abstract}
Mental health problems related to COVID-19 in the health personnel and the general population of Peru.
\end{abstract}
Erick Huertas-Gonzales 1,a,b

Sres. Editores:

La COVID-19 también conocida como la enfermedad del coronavirus tipo 2 del síndrome respiratorio agudo grave (SARS COV 2), descrita por primera vez en la ciudad de Wuhan (China) durante el mes de diciembre del 2019, y que rápidamente se extendió a nivel mundial, está causando daño a la salud mental (SM) del personal de salud (1).

Ante la difícil situación que se encuentra atravesando el país, el personal de salud como primera línea, enfrenta una ardua labor con la finalidad de combatirlo, pero a la vez se encuentran expuestos a un alto riesgo de ser contagiados, discriminados, pasar al aislamiento, a la ausencia o pérdida de contacto con sus familiares y al agotamiento tanto físico como mental, con la posibilidad de padecer problemas de salud mental como la ansiedad, el estrés, insomnio, temor y síntomas depresivos (2).

Dichos problemas, pueden afectar la calidad de atención que brinda el profesional, en sus habilidades para la toma de decisiones, lo que podría generar alguna dificultad en la lucha contra la infección por la COVID-19, así como tener un efecto negativo en su bienestar y su calidad de vida (3).

Por tales motivos, es importante que la salud mental de nuestro personal de salud, sea protegido para controlar no solo la epidemia sino también al personal que está en primera línea (3).
Huang et al., en un artículo publicado en China en una población de 246 participantes, presenta los hallazgos de un estudio descriptivo transversal con la finalidad de conocer el estado psicológico del personal de salud, aplicando las escalas de autoevaluación para la ansiedad y la escala de autoevaluación para el trastorno de estrés postraumático, teniendo como resultado que el personal de salud tiene una alta incidencia tanto de estrés como ansiedad (3).

Del mismo modo, en la población general se cree que la COVID-19 tiene un gran impacto psicológico en la salud física y mental, caracterizado por temor a ser infectado, insomnio, aumento de ingesta de licor y cigarrillos, trastornos de estrés postraumáticos, ansiedad y a una percepción de perder la salud (4).

Actualmente, en el Perú aún no se cuenta con publicaciones acerca de los problemas de salud mental que estén afectando al personal de salud y a la población general a causa de la COVID-19; sin embargo, esto no quiere decir que esta crisis epidemiológica no pueda afectar la salud mental de dicho personal (5).

Por eso es importante la formación de equipos de salud mental en intervención de crisis, conocer el uso de plataformas digitales, la comunicación vía online, realizar entrevistas por telemedicina y hacer uso de instrumentos breves para la detección de problemas de salud mental y que sean validadas en nuestra población, con la finalidad de ser utilizadas para cualquier desafío que depare a la salud pública del país (6).

Facultad de Medicina, Universidad Ricardo Palma. Lima, Perú.

Estudiante de Medicina

b ORCID: 0000-0002-7863-9627 
En el Perú, se cuenta con 4 instrumentos para la detección de problemas de salud mental que están disponibles para su uso en la población, siendo 2 las que más se utilizan, como son las versiones cortas de la Escala de Psicopatología Depresiva (EPD-4 y EPD6) (6), y la Escala de Ansiedad de Lima de 20 ítems (EAL-20) (7).

Finalmente, en esta pandemia de la COVID-19 es muy importante que todas las personas cumplan con todos los protocolos de bioseguridad, lavarse bien las manos con agua y jabón, usar mascarillas, alcohol, mantener distanciamiento social, evitar exponer a las personas más vulnerables y el Estado debería proponer estrategias para poder evitar el estrés y ansiedad en el personal de salud que está en primera línea.

Conflicto de interés. El autor manifiesta no tener conflicto de interés por lo mencionado en esta comunicación.

Financiamiento: Autofinanciado

\section{Correspondencia:}

Erick Michel Huertas Gonzales

Av. Alfredo Benavides 5440, Santiago de Surco 15039. Lima, Perú

Correo electrónico: erik_tauro_1994@hotmail.com

\section{REFERENCIAS BIBLIOGRÁFICAS}

1. Escobar G, Matta J, Taype-Huamaní W, Ayala R, Amado J. Características clínico epidemiológicas de pacientes fallecidos por COVID-19 en un hospital nacional de Lima, Perú. Revista de la Facultad de Medicina Humana. 2020; 20(2): 1-1. DOI 10.25176/ RFMH.v20i2.2940
2. Kang L, Li Y, Hu S, et al. The mental health of medical workers in Wuhan, China dealing with the 2019 novel coronavirus. Lancet Psychiatry. 2020; 7(3):e14. doi: 10.1016/S2215-0366(20)30047-X

3. Huang JZ, Han MF, Luo TD, Ren AK, Zhou XP. Mental health survey of 230 medical staff in a tertiary infectious disease hospital for COVID-19. Zhonghua Lao Dong Wei Sheng Zhi Ye Bing $\mathrm{Za}$ Zhi. 2020;38(0):E001. doi: 10.3760/ cma.j.cn121094-20200219-00063

4. Shigemura J, Ursano RJ, Morganstein JC, Kurosawa M, Benedek DM. Public responses to the novel 2019 coronavirus (2019-nCoV) in Japan: Mental health consequences and target populations. Psychiatry Clin Neurosci. 2020;74(4):281-282. doi: 10.1111/pcn. 12988

5. Brooks SK, Webster RK, Smith LE, et al. The psychological impact of quarantine and how to reduce it: rapid review of the evidence. Lancet. 2020;395(10227):912-920. doi: 10.1016/S01406736(20)30460-8

6. Vega-Dienstmaier JV. Construcción de versiones cortas de la Escala de Psicopatología Depresiva para tamizaje de depresión mayor y sus características psicométricas. Rev Neuropsiquiatr. 2018; 81(3):154164. DOI: $10.20453 /$ rnp.v81i3.3383

7. Lozano-Vargas A, Vega-Dienstmaier JV. Construcción y propiedades psicométricas de la Escala de Ansiedad de Lima de 20 ítems (EAL-20). Rev Neuropsiquiatr. 2018; 81(4):226-234. DOI: 10.20453/rnp.v81i4.3437

Recibido: 26/10/2020

Aceptado: 26/04/2021 